\title{
Nucleated Red Blood Cell
}

National Cancer Institute

\section{Source}

National Cancer Institute. Nucleated Red Blood Cell. NCI Thesaurus. Code C73125.

Red blood cells that are at the last stage of development, still containing a nucleus, that are found in the bone marrow and occasionally in the peripheral blood. 